\title{
THE INFRARED SPACE OBSERVATORY (ISO):
}

\author{
Overview and Status
}

\author{
M.F. KESSLER \\ ISO Science Operations Centre, Astrophysics Division of ESA, \\ VILSPA, P.O. Box 50727, 28080 Madrid, Spain.
}

\section{Introduction}

The Infrared Space Observatory $\left(\mathrm{ISO}^{1}\right.$ ) is a cryogenically-cooled satellite giving the astronomical community, for the first time, the capability of making detailed observations at infrared wavelengths $(2.5-240 \mu \mathrm{m})$ with a sophisticated and sensitive set of observatory-class instruments for a period in excess of two years.

The proposal for ISO was made to ESA in 1979 and it was selected in 1983 to be the next new start. The instruments were chosen in 1985 and ISO's main industrial development started in 1988. Launch took place in November 1995 and the helium coolant is expected to last until April 1998. The calibration and data analysis will be improved during the post-operations phase, which is funded to the end of 2001 .

\section{Mission Overview}

The ISO satellite (Kessler et al. 1996) carries a large cryostat which -at launch-contained about 2300 litres of superfluid helium to maintain the telescope, the scientific instruments and the optical baffles at temperatures between $2 \mathrm{~K}$ and $8 \mathrm{~K}$. Its Ritchey-Chrétien telescope has a 60 -cm diameter primary mirror. A pointing accuracy at the arc second level is provided by a three-axis-stabilisation system consisting of reaction wheels, gyros and optical sensors.

ISO has four scientific instruments, namely: an imaging photo-polarimeter (ISOPHOT, 2.5$240 \mu \mathrm{m}$, Lemke et al. 1996), a camera (ISOCAM, 2.5-17 $\mu \mathrm{m}$, Cesarsky et al. 1996), a short wavelength spectrometer (SWS, 2.5-45 $\mu \mathrm{m}$, de Graauw et al. 1996) and a long wavelength spectrometer (LWS, 43-197 $\mu \mathrm{m}$, Clegg et al. 1996). These instruments were built using national funding by international consortia of scientific institutes, each headed by a single Principal Investigator. However, the instruments were designed to form a complete, complementary and versatile package. As the instruments view slightly different parts of the sky (separated by either $12^{\prime}$ or $17^{\prime}$ ), only one is operational in prime mode at a time; however, ISOCAM and LWS gather additional data in 'parallel' modes and -in as many of the satellite slews as possible- a long-wavelength channel of ISOPHOT is used to make a partial sky survey in a spectral band around $175 \mu \mathrm{m}$.

With ISO, photometry is possible in broad and narrow spectral bands across its entire wavelength range of $2.5 \sim \sim 240 \mu \mathrm{m}$. A variety of apertures, ranging from $5-180^{\prime \prime}$, is selectable out to $120 \mu \mathrm{m}$. For spectroscopy, resolving powers ranging from 50 to 30,000 are available in the wavelength range from 2.5 to nearly $200 \mu \mathrm{m}$. ISO is capable of direct imaging in broad and narrow spectral bands across its complete wavelength range at spatial resolutions ranging from 1.5" (at the shortest wavelengths) to $90^{\prime \prime}$ (at the longer wavelengths). In addition, mapping may be carried out using sequences of pointings.

The operational orbit is highly elliptical with a period of nearly 24 hours, an apogee of $70000 \mathrm{~km}$ and a perigee of $1000 \mathrm{~km}$. For reasons of safety and straylight control, the satellite may not observe close to the Sun, Earth and Moon; thus, at any instant, only around $15 \%$ of the sky is available.

\footnotetext{
'ISO is an ESA project with instruments funded by ESA Member States (especially the PI countries: France, Germany, the Netherlands and the United Kingdom) and with the participation of ISAS and NASA.
} 
Operations are carried out from ESA's satellite tracking station in Villafranca, Spain. In keeping with ISO's rôle as an observatory, over half of its observing time has been made available to European, Japanese and US astronomers via the traditional route of proposals and peer review.

\section{In-orbit status}

ISO was given a perfect launch by an Ariane 44P vehicle during the night of 16-17 November 1995 . During the following 2.5 months, all the satellite systems were successfully verified and the core calibrations of the instruments carried out. Routine operations (i.e. carrying out the planned open and guaranteed time observations) started on 4 February 1996.

The cryogenic system is providing all the expected temperatures. Current estimates, based on two direct measurements of the mass of liquid helium remaining in the tank, are that ISO's in-orbit lifetime will be $\sim 28$ months, well above the requirement of 18 months. This extended lifetime is particularly important as it permits two visits to the Taurus and Orion regions, which were not within ISO's visibility during the nominal mission.

Images of point sources have been made with ISOCAM clearly showing up to the sixth Airy diffraction ring. Analysis of the data has shown that the ISO telescope is diffraction-limited down to a wavelength at least of $15 \mu \mathrm{m}$. Overall the optical performance meets, or exceeds, its specifications.

The pointing performance is also substantially better than the specifications in all aspects. The short term jitter is about $0.5^{\prime \prime}$ ( $2 \sigma$, half cone over a 30 second period) as compared to the specification of $\leq 2.7^{\prime \prime}$. The blind pointing is about $3.5^{\prime \prime}(2 \sigma$, half cone) as compared to the specification of $\leq 11.7^{\prime \prime}$.

The scientific instruments are all operating very well (see fig. 1 and following four papers). The biggest in-orbit issue has been the effects on the sensitivity of the instruments caused by impacts of high-energy cosmic ray particles on the infrared detectors; these necessitated changes in operating conditions and data processing. All instruments are returning scientific data of excellent quality as is shown by the papers in this volume.

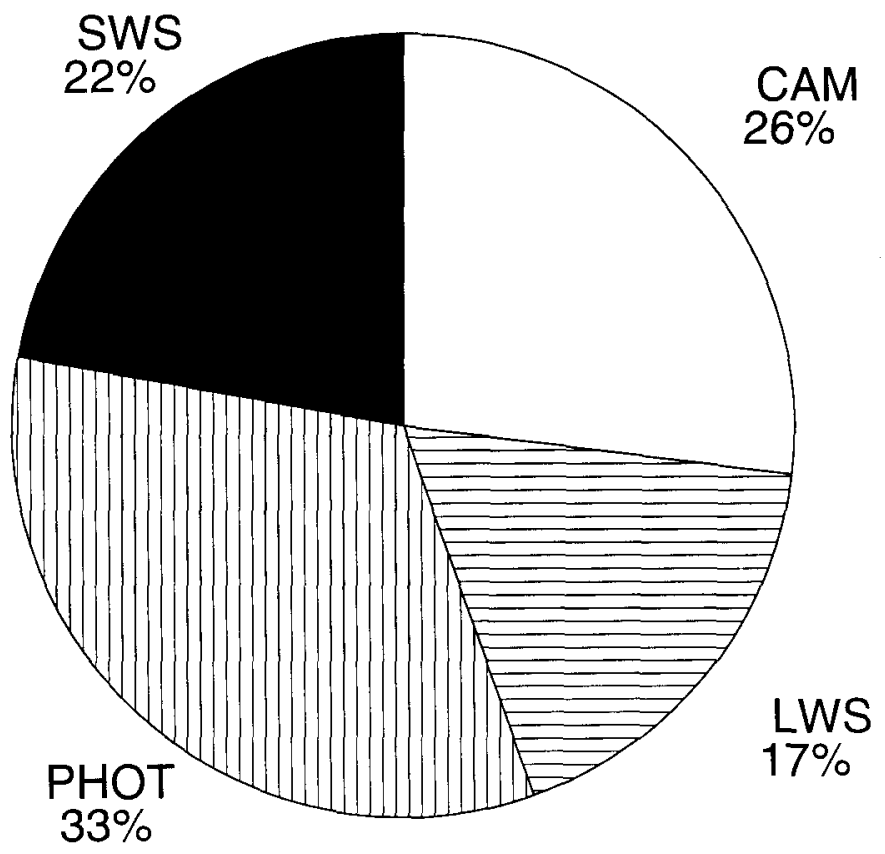

Figure 1. Instrument usage by time 


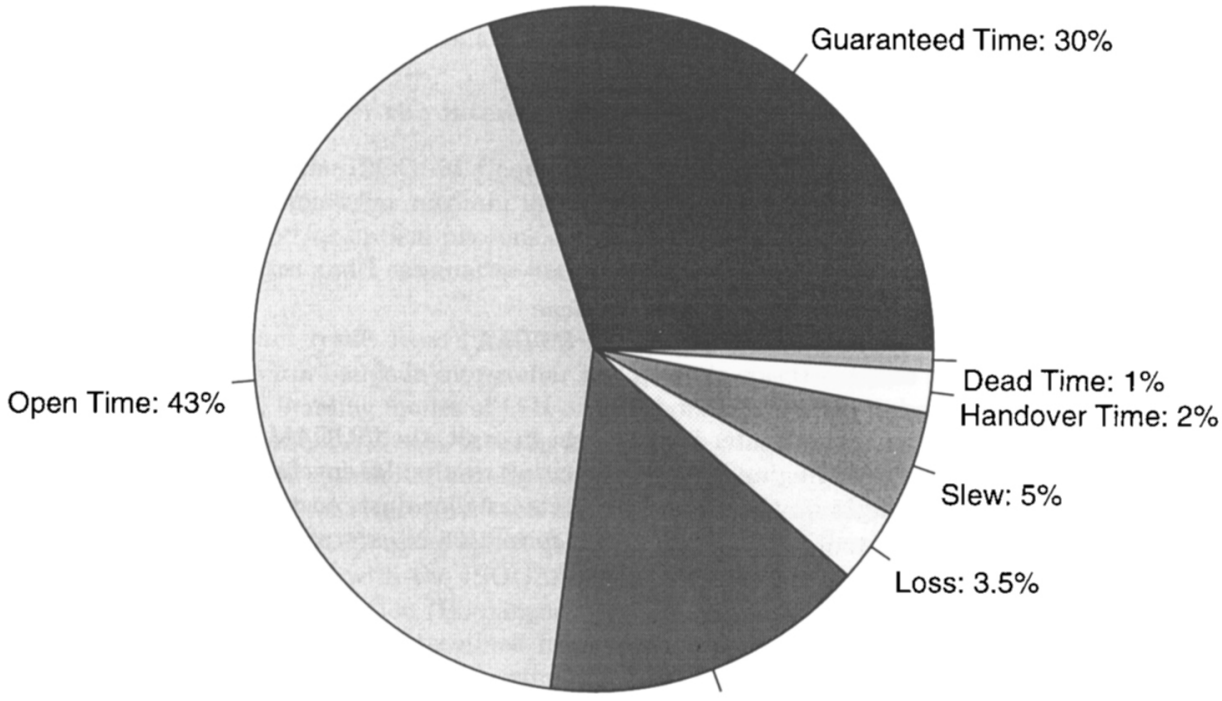

Calibration: $15.5 \%$

Figure 2. Use of ISO time

ISO's ability to investigate an object in depth with all its instruments was was demonstrated early in the mission with the performance verification observations of the Antennae galaxies, a prototypical interacting system (Fischer et al. 1996, Klaas et al. 1997, Kunze at al. 1996, Vigroux et al. 1996)

The ground segment is working very smoothly; efficient observing schedules with highly-graded observations are being regularly planned and executed. Per day, an average of about 40 observations is made, using between $90 \%$ and $95 \%$ of the available time (fig. 2). The remaining time is mainly used for slews and engineering activities. As of August 1997, over 19000 individual observations for nearly 500 observers had been successfully carried out. The data is dispatched to observers on CD-ROMs within 2 weeks of the observation being made.

A network of 7 data centres is being planned to provide support to the community in working with ISO data after the end of the in-orbit mission. An initial archive, with homogeneouslyprocessed data will be put on line at the ISO Data Centre in Villafranca as the proprietary rights periods for the data expire. Some 2-3 years later, all data will be re-processed with improved techniques and calibration files to produce a 'legacy' archive.

In conclusion, the ISO mission is in excellent condition and is performing much better than specifications in most areas. The impressive flow of data is expected to continue until about April 1998, some 10 months more than the requirement.

\section{References}

Cesarsky C.J., Abergel, A., Agnèse, P. et al., 1996, A\&A 315, L32.

Clegg P.E., Ade P.A.R., Armand C. et al., 1996, A\&A 315, L38.

Fischer, J., Shier, L.M., Luhman, M.L. et al., 1996, A\&A 315, L97.

de Graauw, Th., Haser, L.N., Beintema, D.A. et al., 1996, A\&A 315, L49

Kessler, M.F., Steinz, J.A., Anderegg, M.E. et al., 1996, A\&A 315, L27.

Klaas, U., Haas, M., Heinrichsen, I., Schulz, B., 1997, A\&A in press.

Kunze, D., Rigopoulou, D., Lutz, D. et al., 1996, A\&A 315, L101

Lemke D., Klaas, U., Abolins, J. et al, 1996, A\&A 315, L64.

Vigroux, L., Mirabel, F., Altieri, B. et al., 1996, A\&A 315, L93. 